\title{
Los otros Álbumes para la juventud de Robert Schumann ${ }^{1}$
}

\section{The Other Albums for the Young by Robert Schumann}

\section{Os outros Álbuns para a Juventude de Robert Schumann}

\author{
(iD) Virginia Sánchez Rodríguez² \\ Universidad de Castilla-La Mancha, Centro de Investigación y Documentación Musical, \\ Ciudad Real, España \\ virginia.sanchez@uclm.es
}

Resumen: El Album für die Jugend (Álbum para la juventud) Op. 68 de Robert Schumann es una de sus composiciones más conocidas, en parte, debido a su perfil pedagógico y su actual visibilidad en el currículo en los planes de estudios musicales reglados de la especialidad de piano. Ahora bien, a pesar de la existencia de numerosos antecedentes académicos sobre él, hemos podido constatar la existencia de otros volúmenes que, bajo el mismo nombre de Álbum para la juventud, fueron publicados tras el fallecimiento del compositor y sobre los que no hemos localizado trabajos previos. Atendiendo a esta circunstancia, la intención de este trabajo, además de proponer un acercamiento al origen y la esencia del opus 68, comprende conocer las características y el contexto en que estos otros Álbumes para la juventud fueron gestados y comercializados, todo ello a través del acceso a fuentes primarias y secundarias. Como se podrá comprobar, debido a su carácter póstumo, la figura del editor

\footnotetext{
1 En homenaje a Isabel Guerras (1935-2015), virtuosa del piano y maestra entrañable, que me enseñó a amar el Álbum para la juventud de Robert Schumann y tantas otras composiciones.

2 Doctora en Musicología por la Universidad de Salamanca (2013), Virginia Sánchez Rodríguez es Profesora Ayudante Doctora de la Universidad de Castilla-La Mancha e investigadora del Centro de Investigación y Documentación Musical (CIDoM)-Unidad Asociada al CSIC. Es autora de cinco libros y de más de un centenar de artículos de investigación y capítulos de libro en las principales revistas y editoriales. Asimismo, ha impartido más de sesenta ponencias en congresos nacionales e internacionales. Miembro de varios Proyectos I+D+i en convocatorias competitivas nacionales y regionales, es, desde 2016, secretaria y editora de la revista Cuadernos de Investigación Musical, así como coordinadora de los monográficos anuales de música de ArtyHum: Revista Digital de Artes y Humanidades. Entre las becas de investigación recibidas, destacan la Beca de Investigación Etnográfica "Ángel Carril" del Instituto de las Identidades de la Diputación de Salamanca (2016) y la Beca de Investigación en Folklore de CIOFF y el INAEM (2018). Entre los galardones recibidos, destacan el Premio de Investigación a la Mejor Tesis Doctoral de la Fundación SGAE (2013), el I Premio de Investigación "Rosario Valpuesta" (2015) y el Accésit del Premio Internacional de Investigación “Victoria Kent" (2018).
} 
cobró una relevancia fundamental, utilizando el inmenso éxito de la composición schumanniana y su propia experiencia en la edición de este tipo de álbumes.

Palabras clave: Robert Schumann; Álbum para la juventud; literatura pianística; edición musical.

Abstract: The Album für die Jugend (Album for the Youth) Op. 68 by Robert Schumann is one of his best-known compositions, partly according to its pedagogical profile and its visibility in the curriculum in the regulated musical curricula of piano studies. However, despite the existence of numerous academic antecedents about it, we have verified the existence of other volumes that, under the same name of Album for the Youth, were published after the death of the composer, despite the fact that we have not located previous studies about them. According to this circumstance, the objective of this work, in addition to proposing an approach to the origin and essence of opus 68, includes knowing the characteristics and context in which these other Albums for youth were created, all that throughout the access to primary and secondary sources. As we show, due to its posthumous nature, the figure of the publisher gained fundamental relevance, using the immense success of the Schumannian composition and his own experience in publishing this type of album.

Keywords: Robert Schumann; Album for the Youth; pianistic literature; musical edition.

Resumo: O Album für die Jugend (Álbum para a Juventude) Op. 68 de Robert Schumann é uma das suas composições mais conhecidas, em parte de acordo com o seu perfil pedagógico e a sua visibilidade atual nos currículos musicais da especialidade de piano. No entanto, apesar da existência de numerosos trabalhos acadêmicos sobre ele, pudemos constatar a existência de outros volumes que, sob o mesmo nome de Álbum para a Juventude, foram publicados após a morte do compositor, sobre os quais não localizamos trabalhos anteriores. Diante desta circunstância, a intenção deste trabalho, além de propor uma abordagem sobre a origem e essência do opus 68, passa por conhecer 
as características e o contexto em que foram criados esses outros Álbuns para a Juventude, tudo por meio do acesso a fontes primárias e secundárias. Como iremos demonstrar, pelo seu carácter póstumo, a figura da editora ganhou relevância fundamental, valendo-se do imenso sucesso da composição schumaniana e da sua própria experiência na edição deste tipo de álbum.

Palavras-chave: Robert Schumann; Álbum para a Juventude; literatura de piano; edição de música.

Submetido em: 13 de setembro de 2020

Aceito em: 15 de dezembro de 2020 


\section{Introducción}

El Álbum para la juventud Op. 68 de Robert Schumann (18101856) forma parte del actual currículo de los primeros cursos de piano en los estudios musicales reglados de medio mundo. Una de las razones de su éxito es su calidad musical, la belleza de las melodías y sus posibilidades didácticas. Al fin y al cabo, el propio autor concibió la composición como una obra para poder interpretarse en el hogar y destinada, originalmente, a la práctica instrumental de sus vástagos, de ahí el evidente carácter pedagógico.

Además de su presencia educativa, esta composición ha sido, en las últimas décadas, objeto de varios estudios, siendo las contribuciones de Appel (1994, pp. 171-202), Kok (2006, pp. 111-132) y Deahl (2001, pp. 25-42), entre otras ${ }^{3}$, los antecedentes académicos más significativos. En todos ellos-junto a las menciones al opus 68 que salpican las principales monografías sobre su autor (TODD, 1994; JENSEN, 2001; PERREY, 2007; TURNBRIDGE, 2007; KOK Y TUNBRIDGE, 2011; GECK, 2013)- se profundiza en su origen, en su esencia, en el valor interdisciplinar de la música para niños y también en el concepto de Hausmusik que aparece inherente a la obra. Y, de todos los antecedentes, Robert Schumanns "Album für die Jugend": Einführung und Kommentar (APPEL, 2010 -1 ${ }^{\text {a }}$ ed. 1998-) ocupa un lugar esencial no solo por acoger, como parte del primer apéndice, el facsímil de la edición de 1850 sino, además, por ser, hasta la fecha, la más extensa y detallada aportación sobre esta composición.

Ahora bien, conviene señalar que este conocido opus 68 no es la única composición que Robert Schumann escribió encaminada a la juventud con un valor pedagógico y tampoco es la única que, tras su primera edición, fue comercializada bajo el título Álbum para la juventud. La localización de un volumen titulado Zweites Album für die Jugend de Schumann en una colección privada en España fue el

3 De acuerdo con sus aportaciones en torno al valor de la pedagogía del Álbum para la juventud, véase también Gustems, Chaib, Font, et ál., 2007, pp. 49-54. 
punto de partida de esta investigación, que presenta la existencia de otras composiciones en las que Robert Schumann planteó su preocupación por las jóvenes generaciones de músicos y que, tras su fallecimiento, fueron presentadas bajo ese mismo nombre.

En concreto, el principal objetivo de este artículo, además de realizar un acercamiento a las características de los diferentes volúmenes editados como Álbum para la juventud, persigue comprender las circunstancias que rodearon la edición de las diversas obras comercializadas bajo ese título y los principales rasgos de las mismas. Para llevarlo a cabo, este trabajo ha continuado una metodología de carácter cualitativo que se ha visto determinada por las fuentes utilizadas. Aunque accederemos a fuentes secundarias bibliográficas -relativas a los antecedentes académicos más significativos relacionados, especialmente, con el mencionado Op.68-, esta investigación se vertebra, principalmente, en torno a fuentes primarias formadas por varias ediciones de los diferentes Álbumes para la juventud publicados en los siglos XIX y $X X$, el catálogo de la editorial Schuberth correspondiente con las primeras ediciones de las composiciones de Schumann y diversos ejemplares de la prensa alemana de la época.

\section{El Álbum para la juventud Op. 68: renovando la literatura pianística para niños}

En el año 1848, como regalo para el séptimo cumpleaños de su hija mayor, Marie (1841-1929), Robert Schumann escribió un conjunto de piezas sencillas, el germen de lo que, posteriormente, sería el Álbum para la juventud Op. 68. Frente a las obras que el músico había abordado hasta el momento -entre las que destaca su legado pianístico, en parte, porque "para Schumann, o piano tornou-se seu confidente, em que sua produção sonora foi a imagem de um mundo interior conturbado, com buscas de sonoridades, de polirritmias e de polifonías" (FUCCI AMATO, 2006, p. 83)-, las razones que llevaron al compositor a concebir la que 
Los otros Álbumes para la juventud de Robert Schumann

Virginia Sánchez Rodríguez

sería, con el tiempo, una de sus composiciones más conocidas tenía que ver con el tipo de repertorio que solía interpretarse durante los primeros años del estudio del piano. En aquel momento, Robert y Clara Schumann (1819-1896) tenían cuatros hijos, y, de ellos, sus hijas mayores, Marie y Elise (1843-1928), estaban aprendiendo a tocar el piano (DEAHL, 2001, p. 31). El hecho de que los métodos utilizados en la época estuvieran tan encaminados a las cuestiones mecánicas y carecieran de un sentido estético ${ }^{4}$ hegemónico inspiró a Robert Schumann a componer un repertorio propio para su familia. Así lo plasma Clara Schumann en su diario del día 1 de septiembre de 1848, del que se hace eco Bernhard Appel: "pieces children usually study in piano lessons are so poor that it occurred to Robert to compose and publish a volume (a kind of album) consisting entirely of children's pieces" (APPEL, 1994, p. 171).

Días después, entre el 2 y el 27 de septiembre (DEAHL, 2001, p. 32), Schumann amplió el Álbum obsequiado con nuevas piezas, añadiendo canciones del imaginario tradicional y melodías procedentes de piezas ya popularizadas por músicos de la época. Este nuevo proyecto compositivo surgió, por tanto, de una necesidad familiar, un rasgo que lo vincula con Johann Sebastian Bach (1685-1750) en torno a su labor compositiva y recopiladora para el Notenbüchlein für Anna Magdalena Bach (Album para Ana Magdalena Bach), músico al que el propio Schumann admiraba ${ }^{5} \mathrm{y}$ del que consideraba necesario el estudio diario de sus preludios y fugas: "Toca con aplicación las fugas de los buenos maestros, ante todo de Juan Sebastián Bach, El clave bien temperado ha de ser tu pan de cada día. Con él te convertirás en un verdadero músico" (SCHUMANN, 1986, p. XVI). Al fin y al cabo, la relación entre la vida y el arte, entre lo personal y lo profesional, si bien está presente en el legado de todos los compositores, aún encuentra un sentido mayor en torno a Schumann, como señala Alexandre Dias de Oliveira Alcantara:

4"Schumann was concerned about the poor quality of pedagogical piano available music available for teaching his own young daughters. Therefore, in the final decade of his life, he began composing works aimed at satisfying the escalating middle-class demand for Hausmusik" (DEAHL, 2001, p. 25).

5 "It was only in 1829, in fact, that the qualities of Bach's music began to dawn on Schumann; up to that point he had, by his own admission, been unreceptive to both Bach and Goethe. Thereafter his reverence for Bach never faltered" (MARSTON, 2007, p. 56). 
Ahí es donde Schumann se destaca de otros compositores: en que cualquier aspecto de su experiencia vital -lugares, personas, objetos- puede convertirse en material para su arte. Lo prosaico puede transmutarse en poético, y ésta es para Schumann la función del arte. Su música no es el producto de un genio que supuestamente conecta con lo divino, lanzando partituras desde el Olimpo a los mortales, sino que está llena de vivencias que son comunes a todos. Y la influencia es recíproca, es decir, Schumann llena su arte de vida y quiere que su vida esté impregnada de arte (DIAS DE OLIVEIRA ALCANTARA, 2017, p. 58).

Las melodías que conforman el Álbum para la juventud de Schumann, concebidas en un primer momento, por tanto, como "training pieces" (DAVERIO, 2007, p. 74), iban dirigidas, si no a sustituir las lecciones de los tediosos métodos de la época -que, generalmente, solían desmotivar a los neófitos debido a la ausencia de prioridades artísticas-, a que pudieran combinarse con ellas de forma paralela al disfrute de los pequeños intérpretes. Y es que, tal como expone MacDonald, la belleza y la necesidad de despertar interés en el repertorio por parte del intérprete formaba parte del proceso de estudio de una obra musical para Schumann:

In Schumann's view the performer works through a natural process whereby after a first stage infatuation with a work fades, in a second stage concentration on its mechanics takes priority. Only then, in a final, third stage, are the two approaches united in an ideal, artistic presentation (MACDONALD, 2002, p. 537).

Todo ello fue tenido en cuenta en esta colección de melodías que, además de lo mencionado, presenta un valor extramusical en torno a los títulos que las acompañan. Los títulos poéticos atribuidos a cada una de las piezas que conforman este políptico musical cobran relevancia no solo porque la literatura fue la otra gran pasión de Schumann ${ }^{6}$ sino porque son una parte fundamental

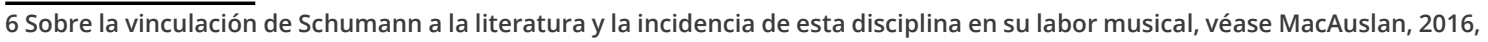
pp. 9-26. 
de la obra desde su concepción original. Debemos recordar que las alusiones poéticas eran habituales en el legado pianístico del músico y que ya habían aparecido desde sus primeras obras, como su Papillons Op. 2 -"the first of Schumann's poetic cycles for keyboard", en palabras de John Daverio (1997, p. 79)- y, conteniendo incluso un título para cada pieza, en su Carnaval Op. 9 -considerado por Peter F. Oswald "a musical picture gallery" (1985, p. 115)-.

En lo que respecta a la composición protagonista de este trabajo, la breve atribución literaria que antecede cada melodía permite añadir significados a la obra por parte del intérprete, pero también por parte del oyente, como señala Kok: "Poetic titles in each movement of the Album imbued the accompanying music with culturally specific meanings and framed performance decisions as well as listeners' expectations" (KOK, 2006, p. 122). Además de su vinculación con la literatura, algunas de las piezas presentan un valor añadido. Es el caso de "Erinnerung", la pieza número 28 del ciclo, que, como señala Carolyn Carrier, está dedicada a Félix Mendelssohn (1809-1847) tras su fallecimiento:

Nearly a year after Mendelssohn had died, the Schumanns still grieved, and Robert composed the wellknown homage to his friend, the "Erinnerung" from the Album für die Jugend, Op. 68. "Erinnerung" did not represent a solitary public tribute to Mendelssohn, however, but rather stood in dialogue with other private album leaves created by the Schumanns and some of their close Friends (CARRIER, 2019, p. 218).

Como Lora Deahl expone, Schumann propuso la publicación de esta composición a la editorial Breitkopf und Härtel bajo el título Weihachtsalbum für Kinder (Christmas Album for Children; Álbum de Navidad para niños) (DEAHL, 2001, p. 32), consciente de las posibilidades comerciales de la Navidad. Sin embargo, la editorial declinó el proyecto y fue entonces cuando, posteriormente, Schumann acudió al editor Julius Schuberth (1804-1875), de 
Hamburgo, que aceptó con mucho gusto, aunque optó por eludir la referencia navideña. En aquel momento, ese primitivo Álbum estaba formado por 42 piezas (APPEL, 2010, p. 188), a pesar de que en su título se indicaba un número menor del siguiente modo: 40 Clavierstïcke für die Jugend von Robert Schumann.

Figura 1. Portada de la primera edición del Álbum para la juventud (1848)

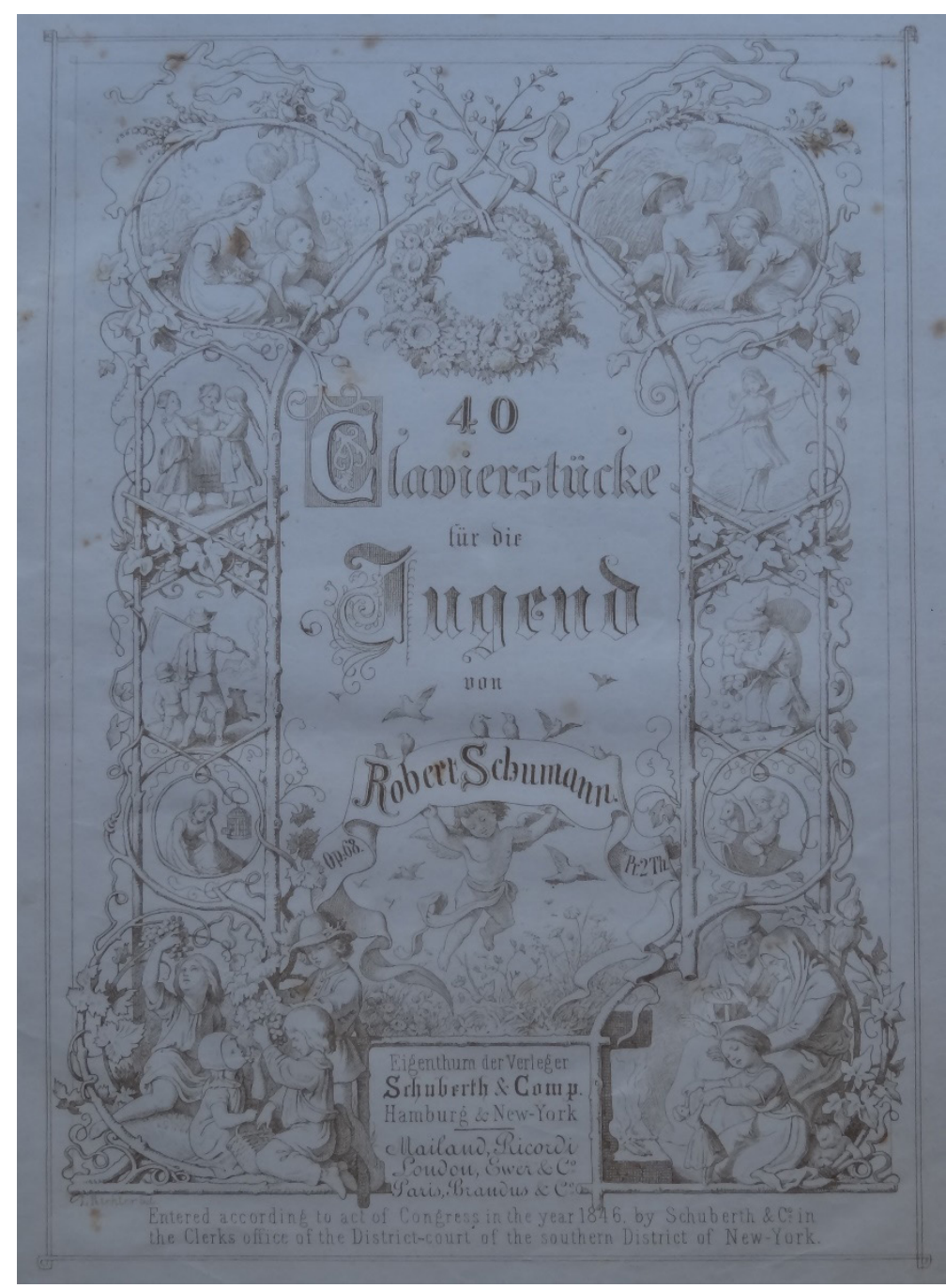

Fuente: Colección Sánchez Rodríguez (España)

Las 42 piezas estaban divididas en dos secciones diferentes dependiendo de la dificultad técnica: la primera sección, "Für Kleinere" ("para los pequeños"), destinada a aquellas creaciones de un nivel más básico, comprendía las piezas 1-18; la segunda 
Los otros Álbumes para la juventud de Robert Schumann

Virginia Sánchez Rodríguez

sección, "Für Erwachsenere" ("para los mayorcitos"), con piezas de una mayor complejidad, estaba formada por los títulos 19-42.

Tabla 1. Distribución de las piezas del Álbum para la juventud (1848)

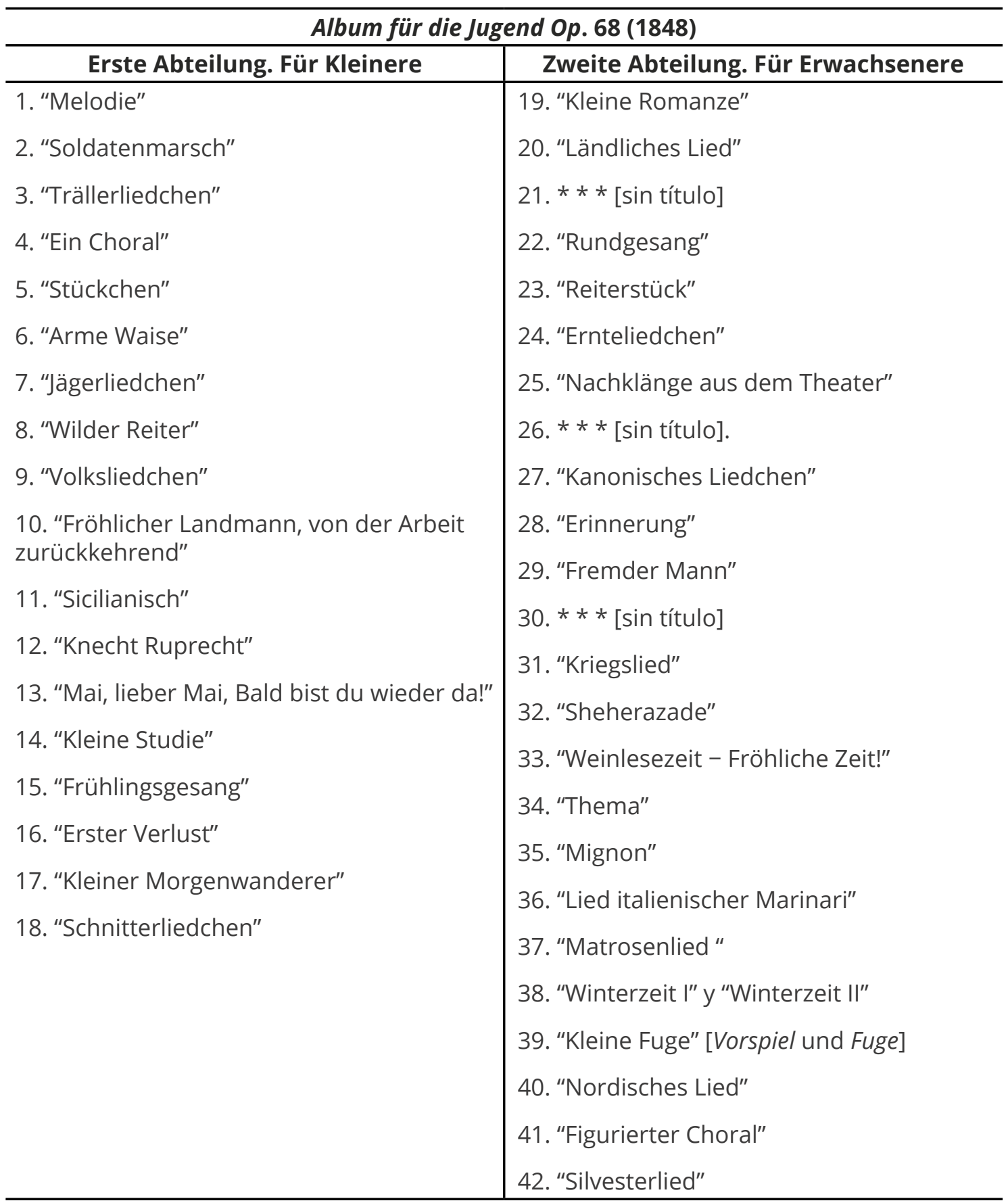


Solo unos meses después de su composición, y tras un ágil trabajo de Schuberth, el Álbum para la juventud fue editado a finales del mismo año 1848 y ya estaba disponible para su venta en enero de 1849, logrando un gran éxito comercial:

\begin{abstract}
Announcements of the publication of the Album first appeared in November and December 1848 in the Neue Zeitschrift für Musik and Signale für die musikalische Welt, and it was finally released to the trade in January 1849. The comercial success of Schumann's enterprise was immediate, widespread, and unprecedent for its time (DEAHL, 2001, p. 35).
\end{abstract}

Si bien es cierto que Robert Schumann ya había compuesto, previamente, obras vinculadas a la juventud, como las célebres Kinderszenen ${ }^{7}$ (Escenas de niños) Op. 15, las diferencias estilísticas y, especialmente, pedagógicas hacen del Álbum toda una novedad. Además, el propio compositor era consciente de las diferencias de su nuevo Op. 68 respecto del Op. 15, tal como expone a su amigo Carl Reinecke (1824-1910), también compositor y pianista, en una carta de 6 de octubre de 1848:

The first thing in "The Album" was written for our eldest child's birthday; and in this way one after another was called forth. It seemed as if I were beginning in my life as a composer anew, and you'll see traces of the old humour. They're decidedly different from the Kinderscenen. Those are retrospective grances by a parent and for grown folks; while "The Christmas Album" contains fore-shadowings, presentiments, and peeps into futurity, for the young (Wasielewski, 1878, p. 259).

Fue tal la acogida del Álbum para la juventud que, en diciembre de 1850, Schuberth, el editor, preparó una segunda edición revisada. En el éxito, además de la belleza de unas melodías sencillas y su valor pedagógico, seguramente tuvo que ver que el conjunto de pequeñas piezas presentaba una renovación del género pianístico para niños:

7 Para una profundización sobre esta obra, véanse, entre otros trabajos: Starobinski, (2002), pp. 361-388; Eigeldinger, 1994, pp. 53-65; Schmidt, 2004, pp. 67-87. 
In writing Op. 68, Schumann not only addressed the necessity of making piano learning pleasurable for children but he also single-handedly created a new genre in piano literature-an album of titled programmatic character pieces written explicitly for children (DEAHL, 2001, pp. 34-35).

\section{Figura 2. Portada de la segunda edición del Álbum para la juventud (1850)}

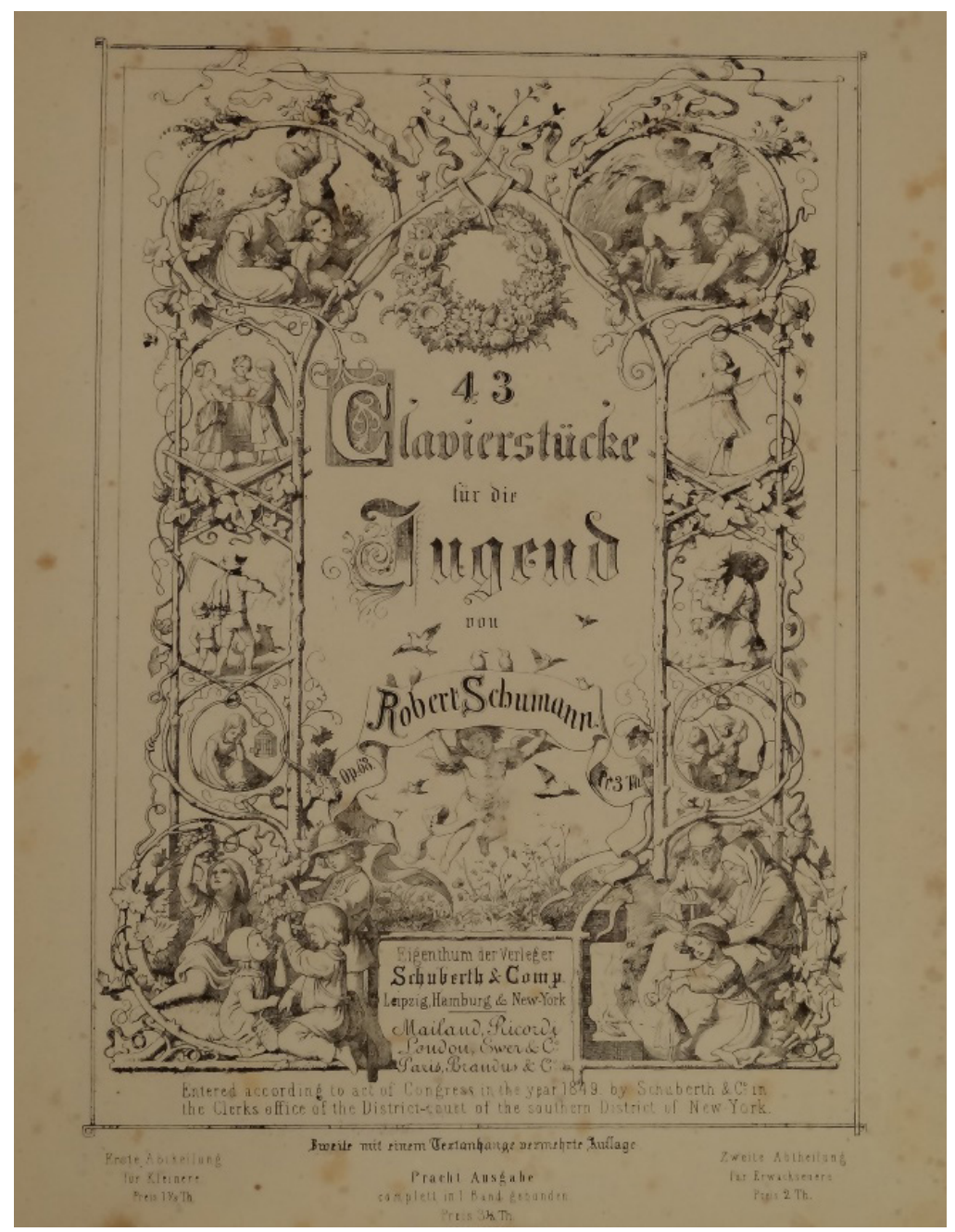

Fuente: Colección Sánchez Rodríguez (España)

En la segunda edición de 1850 se observa, de entrada, una diferencia respecto de la primera versión. En lugar de 42 piezas, el Álbum ahora está formado por 43, algo que no afecta, sin embargo, al contenido musical. Esa diferencia numérica tiene que ver con la división en dos de la pieza titulada "Winterzeit" y que ocupa el 
Los otros Álbumes para la juventud de Robert Schumann

Virginia Sánchez Rodríguez

puesto 38. Así, tanto en esta segunda edición como en las ediciones posteriores hasta la Wiener Urtext Edition, se presentan dos piezas independientes: "Winterzeit I" y "Winterzeit II".

Tabla 2. Distribución de las piezas del Álbum para la juventud (1850)

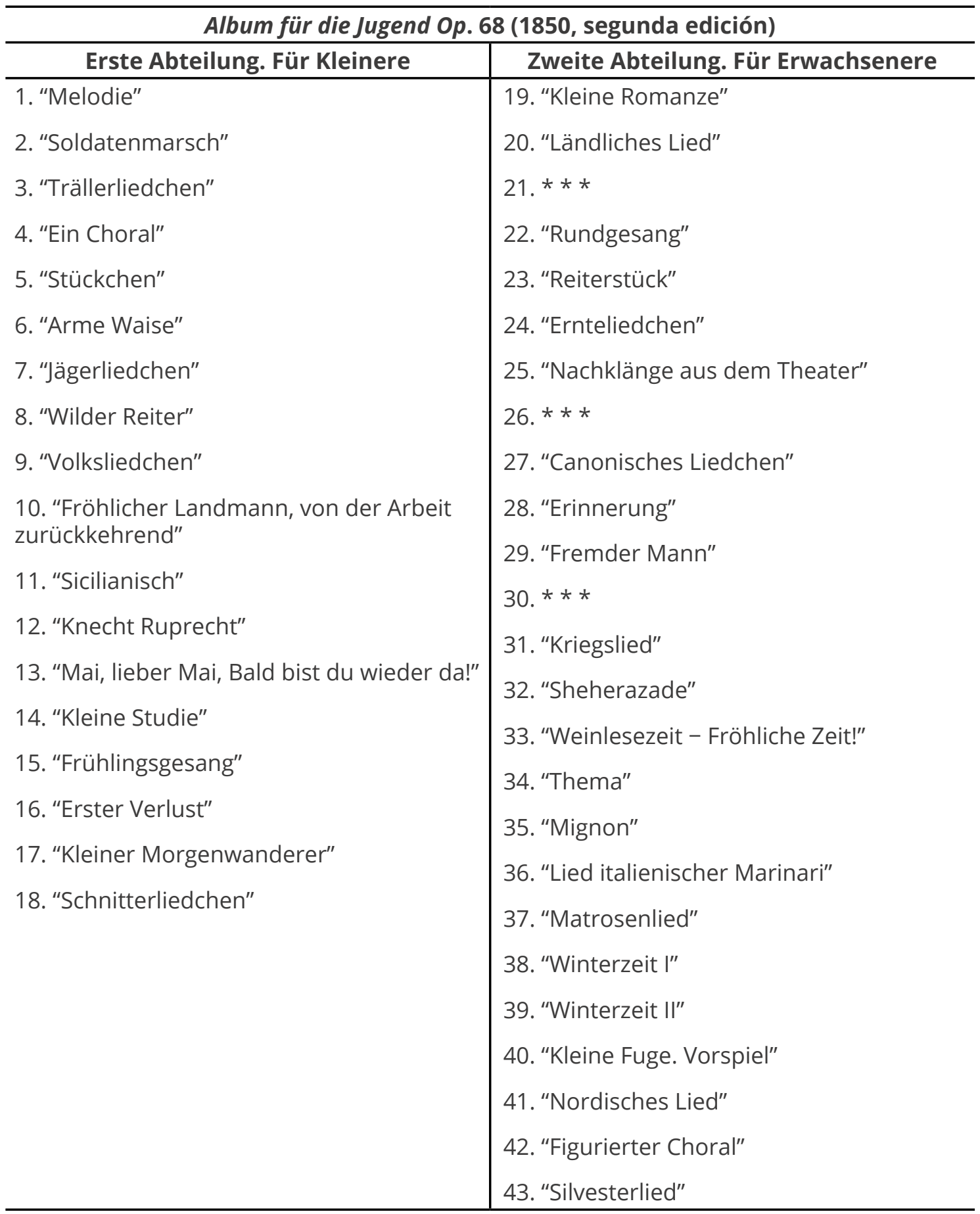


Los otros Álbumes para la juventud de Robert Schumann

Virginia Sánchez Rodríguez

En relación con la sencillez del Álbum, debemos recordar que Schumann siempre defendió la musicalidad por encima de la complejidad técnica sin justificación, frente a algunos compositores románticos que hacían primar un exacerbado virtuosismo basado, principalmente, en cuestiones puramente mecánicas. Ese espíritu habla deuna musicalidad basada en una "interioridad poética", como expone Alexander Stefaniak (2016). Esta filosofía, que entronca con el intimismo y la sencillez, fue defendida por el compositor ya en su "Musikalische Haus-und Lebensregeln" ("Consejos musicales para la casa y para la vida): "Esfuérzate en tocar bien y cuidadamente piezas fáciles: es mejor que ejecutar mediocremente otras más difíciles" (SCHUMANN, 1986, p. XVI). Escritos en 1849 (APPEL, 2010, pp. 194-197) y originalmente publicados en 1850 en el volumen 32 número 36 de Neue Zeitschrift für Musik (1850, 3 de mayo, pp. 1-4) -revista que el propio Schumann había fundado en $1834^{8}$ de acuerdo con sus inquietudes poéticas-, estos "Consejos musicales para la casa y para la vida" posteriormente comenzaron a incluirse como un apéndice del Álbum para la juventud Op. 68 desde esta segunda edición del año 1850. En ellos se refleja el espíritu con el que el propio Álbum había nacido y que lo había convertido en una alternativa o complemento a los métodos pianísticos basados meramente en las cuestiones técnicas:

Debes tocar con aplicación las escalas y otros ejercicios de digitación. Pero hay mucha gente que piensa que con esto pueden alcanzarlo todo, y pasan hasta su vejez haciendo diariamente muchas horas de ejercicios mecánicos. Esto es como si uno se esforzase diariamente en pronunciar el abecedario cada vez más deprisa (SCHUMANN, 1986, p. XVI).

Si bien es cierto que Schumann había compuesto, previamente, piezas que evocaban la niñez -como es el caso de las mencionadas Escenas de niños Op. 15-, la novedad del Álbum para la juventud respecto de su enfoque pedagógico, de acuerdo con la convivencia

8 Para una profundización sobre los orígenes del Neue Zeitschrift für Musik, véase el primer capítulo de Plantinga, 1967. 
de sencillez, belleza y musicalidad, animó al músico a la composición de más piezas dirigidas a público infantil y a principiantes del piano:

Schumann's initial essay in this genre, the Album for the Young (Album für die Jugend), Op. 68, not only revolutionized attitudes concerning music education, but also inaugurated an entirely new genre of piano literature-programmatic music written explicitly for children. The spectacular and instantaneous success of the Album inspired Schumann to write many more pieces for children, spawned a host of copycat publications, and most importantly, popularized a forward-looking pedagogical philosophy whose ramifications extended into the twentieth century (DEAHL, 2001, p. 25).

Es así como nace, por ejemplo, y aun tratándose de piezas vocales, el Liederalbum für die Jugend Op. $79^{9}$ (Album of Songs for the Young, Álbum de canciones para la juventud). Y es que el propio Schumann ya había comprobado el éxito del Op. 68, pero también había reflexionado sobre las posibilidades comerciales de todo tipo de productos dirigidos a los niños:

At the same time, Schumann was fully aware of the commercial possibilities of products marketed for children. Recognition of childhood as a separate developmental phase had spawned profitable cottage industries in the production of children's toys, clothing, furniture, calendars, songs, and books (DEAHL, 2001, p. 32).

Más allá de las propias composiciones de Schumann, esas mismas posibilidades comerciales de lo infantil como seguridad de éxito fueron también aplicadas por su editor, Julius Schuberth, de forma póstuma al compositor, en torno a varias obras publicadas en vida. Y, para ello, habría utilizado el concepto Album für die Jugend

9 Para una profundización sobre la obra, véase Finson, 1990, pp. 227-250. 
Los otros Álbumes para la juventud de Robert Schumann

Virginia Sánchez Rodríguez

como reclamo comercial y sello de garantía, tal como presentamos a continuación.

\section{La creación de una marca comercial: los otros Álbumes para la juventud de Robert Schumann}

En una colección privada ubicada en España se custodia un volumen titulado Zweites Album für die Jugend (SCHUMANN, 1861) (Segundo Álbum para la juventud) de Robert Schumann. De entrada, este ejemplar llamó nuestra atención porque cuenta con una dedicatoria manuscrita de Clara Schumann fechada en el año 1883 y destinada a una mujer llamada Flora Schwarz ${ }^{10}$. Sin embargo, más allá de esa circunstancia, el título de esta partitura despertó nuestro interés por sí mismo debido a que en el catálogo de opus del compositor no aparece ninguna obra con ese nombre. Un acercamiento a la edición localizada permite confirmar que, bajo esa denominación, se engloban dos vestigios del compositor alemán, 12 Klavierstücke für kleine und große Kinder Op. 85 y Neun Ball-scenen Op. 109. Véase al respecto la portada del volumen (Figura 3).

10 Una profundización sobre las circunstancias que rodearon la dedicatoria del volumen por parte de Clara Schumann y la identidad de la receptora de esta obra es recogida en Sánchez Rodríguez, 2021. 
Figura 3. Portada del Zweites Album für die Jugend de Robert Schumann (c. 1861)

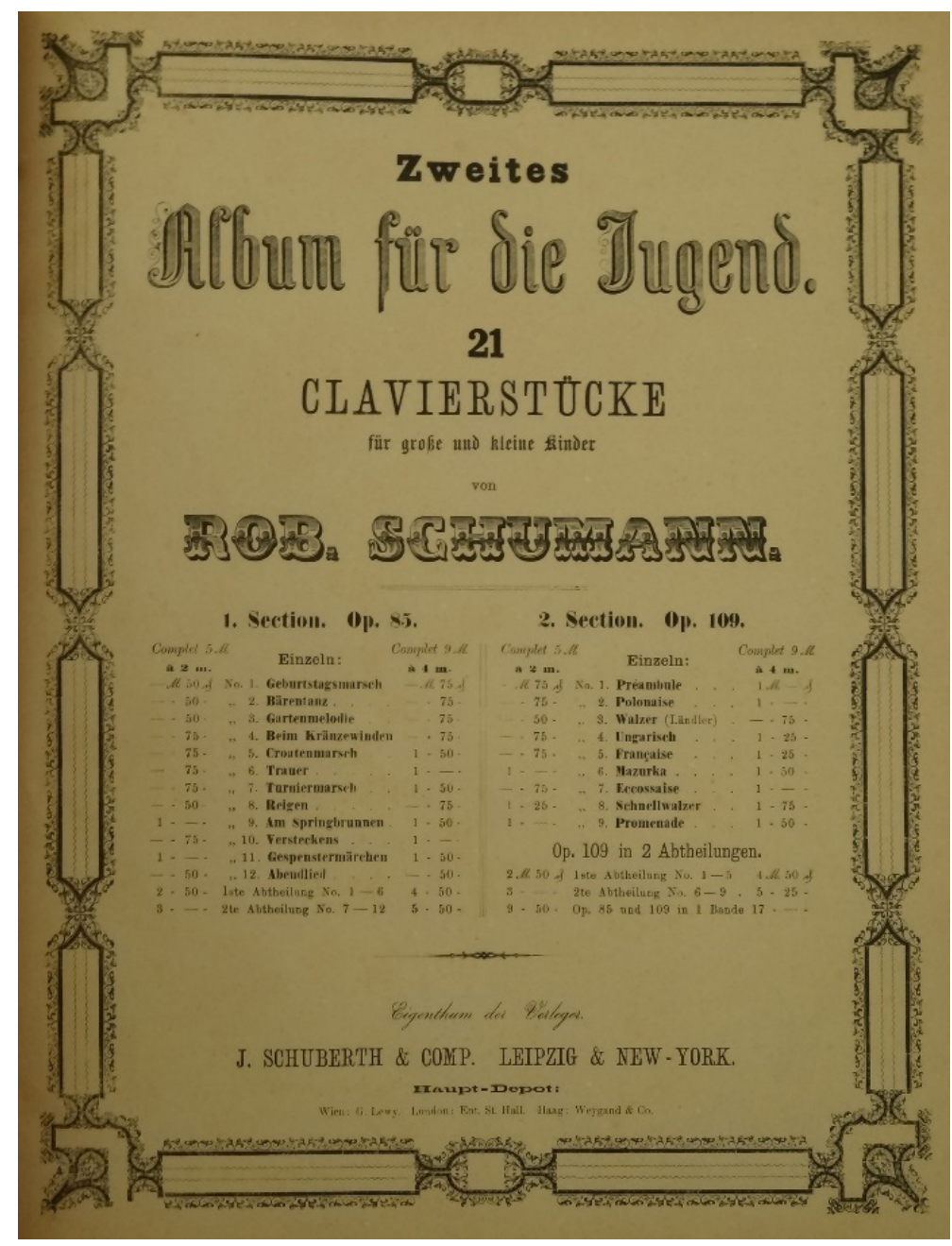

Fuente: Colección Sánchez Rodríguez (España)

Originalmente, las dos obras mencionadas, Op. 85 y $0 p$. 109, fueron escritas para cuatro manos. Esa circunstancia se mantiene en la edición localizada del Segundo Álbum, que contiene ambos opus en dos volúmenes diferentes. El primer volumen, 12 Klavierstücke für kleine und große Kinder (Piezas de piano para niños de todas las edades), contiene una colección de piezas para cuatro manos, de dificultad baja, concebidas para el aprendizaje de las nociones técnicas básicas de forma simultánea al disfrute de las bellas melodías schumannianas, combinando, de este modo, la técnica, la pedagogía y la estética. 
Los otros Álbumes para la juventud de Robert Schumann

Virginia Sánchez Rodríguez

Schumann escribió estas doce melodías en 1849 -tan solo un año después del Álbum para la juventud Op. 68-y una revisión de su contenido permite comprobar que, desde el punto de vista estilístico, se observan numerosas similitudes con las piezas concebidas en el original Op. 68, como la sencillez, un nivel técnico medio-bajo -lo que permite su interpretación por parte de estudiantes que se inician en el estudio musical y de aficionados del piano que quieren disfrutar de la música de cámara a través de las composiciones para cuatro manos- y unas melodías basadas en los principios de la simetría y la belleza. Asimismo, al igual que en el $0 p .68$, Schumann también concibió un título para cada una de las doce piezas que forman parte de este Op. 85.

Por su parte, el segundo volumen del Segundo Álbum para la juventud está dedicado a Neun Ball-scenen (Nueve escenas de baile) Op. 109. Se trata de un conjunto de nueve piezas, también para cuatro manos, que fueron escritas por Robert Schumann en el año 1851. Con una dificultad un tanto más elevada que el Op. 85, en las piezas del Op. 109 se observa un lirismo mayor, fruto de una extensión más amplia y, por tanto, de mayores posibilidades de desarrollo temático, armónico y expresivo para el compositor.

Como Kok señala, el título definitivo fue asignado tras conversaciones con Julius Schuberth y después de barajar la opción de KinderBall11 y Maskentanz (KOK, 2008, p. 113). Finalmente, y a pesar del miedo de Schuberth por la abundancia de los álbumes de danzas que estaban publicándose en la época ${ }^{12}$, se estableció el título Ball-scenen para el "Preámbulo" y las ocho danzas que configuran la composición.

\footnotetext{
11 Originalmente, éste habría sido el título que el compositor se había planteado para la obra: “He originally intended to call the cycle of tone-pieces contained in Op. 109 "The Children's Ball". When he played them through with his wife on their completion, he jocosely interpreted at the "Préambule", "Here the servants pass among the guests with dishes". Farther on he said, "Gradually the grown people mingle in the crowd, and the affair becomes more serious". The composition may afterwards have seemed to him too grave for a "Children's Ball", and he chose the title of "Scenes at a Ball". His creative spirit is here displayed in its most agreeable light. But he did not abandon the idea of a "Children's Ball", and carried it out in 1853" (Wasielewski, 1878, p. 187). Posteriormente, debido al nuevo título para la composición de la que estamos hablando, Schumann decidió utilizar la idea de KinderBall para su Op. 130.

12 "Because of that Schuberth feared the mere presence of the word "Ball" would cause Schumann's work to be received as mere dances for the young?then all the rage. Albums of dance music closely rivaled arrangements of opera melodies in numbers advertised (...). It is remarkable that Schuberth \& Co. seems to have avoided this fad altogether" (KOK, 2008, pp. 116117).
} 
Tabla 3. Distribución de las piezas del Segundo Álbum para la juventud (1861)

\begin{tabular}{|c|c|}
\hline \multicolumn{2}{|c|}{ Zweites Album für die Jugend (1861) } \\
\hline $\begin{array}{l}12 \text { Klavierstücke für kleine und große Kind- } \\
\text { er Op. } 85 \text { (volumen I) }\end{array}$ & $\begin{array}{c}\text { Neun Ball-scenen Op. } 109 \\
\text { (volumen II) }\end{array}$ \\
\hline 1. "Geburtstagmarsch" & 1. "Préambule" \\
\hline 2. "Bärentanz" & 2. "Polonaise" \\
\hline 3. "Gartenmelodie" & 3. "Walzer" \\
\hline 4. "Beim Kränzewinden" & 4. “Ungarisch" \\
\hline 5. "Kroatenmarsch" & 5. "Française" \\
\hline 6. "Trauer" & 6. "Mazurka” \\
\hline 7. “Turniermarsch" & 7. "Ecossaise" \\
\hline 8. "Reigen" & 8. "Walzer" \\
\hline 9. "Am Springbrunnen" & 9. "Promenade" \\
\hline 10. "Versteckens" & \\
\hline 11. "Gespenstermärchen" & \\
\hline 12. "Abendlied" & \\
\hline
\end{tabular}

Se desconoce la fecha exacta de la edición de este Segundo Álbum formado conjuntamente por los opus 85 y 109 debido a que ese dato no aparece impreso en el ejemplar. Sin embargo, la revista Neue Zeitschrift für Musik se hizo eco de la publicación del Zweites Album für die Jugend el 15 de febrero de 1861 (p. 8) como una novedad del momento:

A los numerosos admiradores del brillante compositor, que lamentablementefalleció demasiado pronto, queremosinformarles que su ampliamente difundido Álbum para la juventud ha recibido una secuela, compuesta por su Op. 85, 12 Klavierstücke für kleine und große Kinder, y su Op. 109, Neun Ball-scenen. Charakterstücke.

R. Schumann, Segundo Álbum de la Juventud para pequeños y mayorcitos, en dos volúmenes, para dos manos 1 2/3 thl. cada uno, y para cuatro manos 3 thl. Preciosa edición en un volumen, adornada con la doble imagen de Robert y Clara Schumann, solo 3 $1 / 6$ thl., para cuatro manos $52 / 3$ thl. 
Los otros Álbumes para la juventud de Robert Schumann

Virginia Sánchez Rodríguez

J. Schuberth \& Comp., Leipzig y New York (Neue Zeitschrift für Musik, 15 de febrero de 1861, p. 8$)^{13}$.

El hecho de que se recoja en la prensa en 1961 -y de que, de forma previa, no tengamos constancia de esta edición- podría indicar, seguramente, que esa fue la fecha de lanzamiento de ambos opus juntos bajo el nombre de Segundo Álbum. Podemos imaginar que se esperaba una buena acogida de esta novedad editorial, pues también se preparó una versión para dos manos. Asimismo, en ese mismo número de Neue Zeitschrift für Musik también se confirma que la comercialización de este Segundo Álbum para la juventud se realizó en torno a diferentes formatos de edición. Por un lado, cada una de las piezas, a cuatro manos, era vendida de forma individual. Por otro lado, el Segundo Álbum también se publicó completo en cuatro volúmenes, de manera que cada opus, a cuatro manos, quedaba estructurado en dos ejemplares ${ }^{14}$.

Seguramente, la existencia de este Segundo Álbum para la juventud y su venta en tantos formatos distintos tendría que ver con razones comerciales, de tal forma que se pudiera llegar al mayor número posible de pianistas aficionados. A lo largo de su vida, además de su interés en las cuestiones puramente artísticas, Schumann también se preocupó de algunas cuestiones mercantiles de sus composiciones, como expone Anthony Newcomb (1990, pp. 258-314). A este respecto, su especialización en las composiciones para niños, que es un género en el que, como hemos expuesto anteriormente, ya tenía cierta experiencia, le permitió desarrollar, en palabras de John Daverio, "a more conventional, accessible and commercially successful style" (DAVERIO, 1997, p. 392), lo que podía significar, además, una oportunidad para incrementar las ventas. Ahora bien, conviene apuntar que este Segundo Álbum habría sido publicado, como señalamos, hacia 1861, una fecha posterior al fallecimiento del compositor. Por tanto, la decisión de comercializar los opus 85 y 109 conjuntamente bajo el título de Zweites Album für

13 Traducción de la autora.

14 En el caso del Op. 85, el primer volumen comprendió desde la pieza 1 ("Geburtstagsmarsch") hasta la pieza 6 ("Trauer"), siendo el segundo de ellos el repositorio de las obras comprendidas entre la número 7 (Turniermarsch") y la número 12 ("Abendlied"). En el caso del Op. 109, el primer volumen comprendió de la pieza 1 ("Préambule") a la pieza 5 ("Française"), dejando el segundo volumen para las piezas 6 ("Mazurka"), 7 ("Eccossaise"), 8 ("Schnellwalzer") y 9 ("Promenade"). 
Los otros Álbumes para la juventud de Robert Schumann

Virginia Sánchez Rodríguez

die Jugend corrió a cargo del propio editor, Schuberth, utilizando la marca Álbum para la juventud como un reclamo publicitario.

Llegados a este punto podríamos preguntarnos lo siguiente: ¿existen más Álbumes para la juventud? Roe-Min Kok, en “Negotiating Childrens Music: New Evidence for Schumann's'Charming' Late Style", habla de la existencia de un Tercer Álbum para la juventud (Drittes Album für die Jugend) que estaría formado por la comercialización del Op. 109 en solitario: "In fact, Schuberth \& Co. re-issued Op. 109 as 'Part Three of Album für die Jugend' in 1876" (KOK, 2006, p. 117). Sin embargo, previamente a esa fecha, hemos constatado la existencia de un Tercer Álbum con un contenido diferente.

En el año 1861 se presentan tres álbumes bajo ese nombre. De entrada, como se expone en Neue Zeitschrift für Musik, se habla de un Erstes Album für die Jugend, título al que también hace referencia Appel (2010, p. 193). Este Primer Álbum aparece ahora formado por cincuenta y cinco piezas para dos y cuatro manos que no solo contiene el Op. 68, sino que, bajo la inserción del número ordinal, también alberga las 3 Jugend-Sonaten Op. 118, todo ello organizado en un total de tres volúmenes ${ }^{15}$-los dos primeros, destinados al Op. 68; el tercero, para las mencionadas sonatas-.

El propio Schumann concibió un espíritu juvenil en el origen de estas sonatas Op. 118, que nacieron como Drei Klaviersonaten für die Jugend y que fueron escritas a comienzos del año 1850. Schumann persiguió su vinculación a la juventud a través del título original de las sonatas y quiso recoger un sentido ascendente en lo que respecta a la dificultad, tal como se observa en una carta emitida por Schumann a Schuberth, su editor, en julio de 1853: “I have another work which may interest you. I recently composed three sonatas for young pianists that progress from the very easy to difficult" (KOK, 2008, p. 106). Por tanto, aun tratándose de una obra más académica que otras formas del romanticismo y de mayores dimensiones -cada sonata estaba formada por los habituales cuatro movimientos del clasicismo-, y a pesar de que,

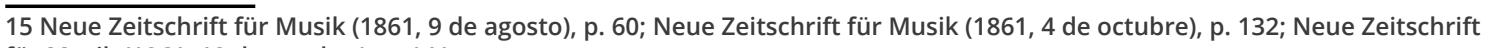
für Musik (1861, 18 de octubre), p. 141. 
Los otros Álbumes para la juventud de Robert Schumann

Virginia Sánchez Rodríguez

según Rosen (1980, p. 315) y Lester (1995, pp. 189-210), las sonatas de Schumann no pueden ser presentadas bajo la premisa de una sonata según su esquema clásico ${ }^{16}$, el compositor tiene presente en todo momento el sentido didáctico, aunque quiere que se evidencien las diferencias de estas tres sonatas respecto del Álbum para la juventud Op. 68. Así parece presentarse en una carta recogida por Roe-Min Kok dirigida al editor: "Here are the 3 sonatas. It [the work] is completely different from the Album. One must familiarize the young also to the performance of larger movements [...]" (KOK, 2008 , p. 118). Estas Sonatas fueron originalmente publicadas para la campaña de Navidad de 1853 en solitario, después de grandes esfuerzos del compositor, que perseguía una publicación lo más rápida posible (KOK, 2008, p. 107).

Ahora bien, ¿por qué las 3 Sonatas para la juventud Op. 118 fueron incluidas por el editor ya en 1861, tan solo cinco años después del fallecimiento de Robert Schumann, como parte del primer volumen de la colección de Álbum para la juventud junto al Op. 68? Como afirma Roe-Min Kok, al parecer, desde el punto de vista comercial, no se lograron los objetivos previstos: "There are signs that Schuberth \& Co. found childrenss sonatas difficult to sell" (KOK, 2008, p. 119). Seguramente, Schuberth pensó en el incremento de las ventas si se adscribían a esta marca publicitaria de Álbum, aunque conviene apuntar que, de forma no oficial, desde el año 1854, el editor ya solía mencionar sus similitudes con la obra pedagógica como un recurso publicitario en la comercialización individual de las sonatas: “These sonatas are a counterpart to Op. 68" (KOK, 2008, p. 119).

También en el año 1861 salió al mercado un Tercer Álbum, Drittes Album für die Jugend, que contenía 38 Lieder-Transcriptionen für gebildete Pianisten (38 Transcripciones de canciones para pianistas capacitados). Así lo recoge Neue Zeitschrift für Musik el día 4 de octubre de 1861 (p. 132). Una mirada al legado schumanniano nos permite observar la ausencia de transcripciones como parte de

\footnotetext{
16 Esa misma idea es planteada por Alexandre Días de Oliveira Alcantara : “En realidad, el problema no es que las piezas de Schumann carezcan de forma, sino que no siguen los patrones esperados por un sector del público todavía muy arraigado en las tradiciones formales del Clasicismo (...). Es importante resaltar que el compositor alemán no desprecia en absoluto la forma, sino que rechaza el concepto, implícito (y con frecuencia, explícito) en las críticas negativas de su obra, de que formas preexistentes sirven como marco universal para todos los compositores, los cuales tendrían entonces que amoldar sus ideas a la forma elegida". Dias de Oliveira Alcantara, 2017, p. 55.
} 
Los otros Álbumes para la juventud de Robert Schumann

Virginia Sánchez Rodríguez

sus opus. En concreto, las obras que forman parte de este Álbum fueron composiciones para voz y piano de Schumann, con las excepciones de la transcripción de un Lieder de Clara Schumann - "Liebeszauber" (6 Lieder, Op. 13 n 3)- y la presencia de dos obras que ya fueron escritas, en su origen, para piano por Robert Schumann -"Romanze" (4 Klavierstücke, Op. 32, n³) y "Abendlied" (12 Klavierstücke für kleine und große Kinder, Op. 85, $\mathrm{n}^{\circ}$ 12)-.

Tabla 4. Distribución de las piezas del Tercer Álbum para la juventud (1861)

\begin{tabular}{|c|c|}
\hline \multicolumn{2}{|r|}{ Drittes Album für die Jugend (1861) } \\
\hline \multicolumn{2}{|r|}{38 Lieder-Transcriptionen für gebildete Pianisten } \\
\hline \multirow[t]{3}{*}{ Volumen I. } & "Die beiden Grenadiere", de Romanzen und Balladen, vol. II, Op. 49 n 1 \\
\hline & "Liebeszauber", de 6 Lieder, Op. 13 n 3 (C. Schumann) \\
\hline & $\begin{array}{l}\text { “Dem roten Röslein gleicht mein Lieb”, de Lieder und Gesänge, vol. I, Op. } \\
27 \mathrm{n}^{\circ} 2\end{array}$ \\
\hline Volumen II & $\begin{array}{l}\text { Gesänge, Op. 31: 1. "Die Löwenbraut"; 2. “Die Kartenlegerin"; 3. “Die } \\
\text { rote Hanne” }\end{array}$ \\
\hline Volumen III & $\begin{array}{l}\text { Lieder, Op. 33: 1. "Der träumende See"; 2. “Die Minnesänger"; 3. “Die } \\
\text { Lotosblume"; 4. "Der Zecher als Doctrinair"; 5. "Rastlose Liebe"; } 6 . \\
\text { "Frühlingsglocken" }\end{array}$ \\
\hline Volumen IV & $\begin{array}{l}6 \text { Gedichte, Op. 36: 1. "Sonntags am Rhein"; 2. "Ständchen"; 3. "Nichts } \\
\text { Schöneres"; 4. "An den Sonnenschein; 5. "Dichters Genesung"; } 6 . \\
\text { "Liebesbotschaft" }\end{array}$ \\
\hline Volumen V & $\begin{array}{l}5 \text { Lieder, Op. 40: 1. "Märzveilchen"; 2. "Muttertraum"; 3. “Der Soldat"; } 4 . \\
\text { "Der Spielmann"; 5. “Verratene Liebe" }\end{array}$ \\
\hline Volumen VI & $\begin{array}{l}3 \text { Gesänge, Op. 83: 1. “Resignation”; 2. “Die Blume der Ergebung”; } 3 . \\
\text { "Der Einsiedler" }\end{array}$ \\
\hline Volumen VII & $\begin{array}{l}3 \text { Gedichte, Op. 119: 1. “Die Hütte”; 2. “Warnung”; 3. “Der Bräutigam } \\
\text { und die Birke” }\end{array}$ \\
\hline Volumen VIII & $\begin{array}{l}5 \text { Heitere Gesänge, Op. 125: 1. "Die Meerfee"; 2. "Husarenabzug”; } 3 . \\
\text { "Jung Volkers Lied"; 4. "Frühlingslied"; 5. "Frühlingslust" }\end{array}$ \\
\hline \multirow[t]{5}{*}{ Volumen IX } & "Sehnsucht", de Lieder und Gesänge, vol. II, Op. 51, nº 1 \\
\hline & "Romanze", de 4 Klavierstücke, Op. 32, n 3* \\
\hline & "Ich wandre nicht", de Lieder und Gesänge, vol. II, Op. 51, n 3 \\
\hline & $\begin{array}{l}\text { "Abendlied", de } 2 \text { Klavierstücke für kleine und große Kinder, Op. 85, } n^{\circ} \\
12^{*}\end{array}$ \\
\hline & * Estas piezas ya fueron concebidas, en su origen, para piano \\
\hline
\end{tabular}

Fuente: elaboración propia. 
Los otros Álbumes para la juventud de Robert Schumann

Virginia Sánchez Rodríguez

La transcripción para piano solo corrió a cargo del pianista y compositor Reinecke-al que, como hemos señalado anteriormente, unía una estrecha amistad a Schumann-y de Auguste Horn (18251893), compositor y arreglista, quien también fue el encargado de la transcripción para cuatro manos del Op. 68 contenida en el Primer Álbum ${ }^{17}$ mencionado de forma previa. El trabajo de transcripción de estas canciones comenzó en 1848 y el propio compositor dio su visto bueno a dicha labor debido a la confianza que tenía en los encargados, especialmente en Reinecke, quien se había ocupado de la transcripción a dos manos de su Op. 85 de una forma temprana ${ }^{18} \mathrm{y}$ a quien le manifestó, de forma específica, su reconocimiento en estas 38 Lieder-Transcriptionen en una carta dirigida el 30 de junio de 1848:

On the whole, I am, as you may suppose, no friend to songtranscriptions; and Liszt's are, for the most part, abominable. But I felt quite easy in your hands, dear Mr. Reinecke, because you understand me as but few do: you only pour the music into another vessel, without peppering and spicing it, a la Liszt. So I like your work, and thank you heartily for it (WASIELEWSKI, 1878, P. 255).

En cuanto a la razón de estas transcripciones, podemos imaginar que, utilizando de nuevo el concepto de Álbum para la juventud en el título como reclamo, el editor quiso comercializar esta selección de Lieder $^{19}$ de Robert Schumann además de una transcripción para piano de Clara Schumann. Recordemos que Clara no solo fue la esposa del compositor (COOPER, 1996, pp. 151158), sino, además, una de las mejores pianistas de toda Europa,

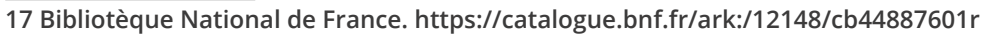

18 Reinecke había sido también el encargado de la transcripción para dos manos del Op. 85 que forma parte del legado de Schumann del catálogo de la editorial Schuberth en 1859, aunque en esta ocasión el mencionado Op. 85 no aparece presentado como Segundo Álbum para la juventud sino, únicamente, como Segundo Álbum (sin vinculación específica a la marca comercial). Así se puede comprobar en las novedades editoriales recogidas en la Neue Zeitschrift für Musik en 1859. Neue Zeitschrift für Musik v. 51, n. 4 (22 de julio), p. 32, 1958.

19 Si bien es cierto que el legado del músico alemán comprende, especialmente, música para piano, durante la década de los años 40 se produce una eclosión de la música para canto y piano, por, entre otras razones, sus posibilidades creativas. Para una profundización sobre la composición de Lieder posterior a 1939, véase Turchin, 1981, pp. 392-404.
} 
Los otros Álbumes para la juventud de Robert Schumann

Virginia Sánchez Rodríguez

sin distinción de género ${ }^{20}$, una temprana compositora y la principal difusora del legado de Robert gracias a su labor performativa pero también a su trabajo como editora de correspondencia y también de algunas de sus partituras ${ }^{21}$, siendo especialmente recordada la edición de la obra completa de su esposo que se publicó en 1879 y $1893^{22}$. A pesar de que Clara Schumann no se ocupó de estas transcripciones, la inserción de una de sus obras en este Tercer Álbum es, sin duda, un reconocimiento a su labor en la producción de su esposo y una valoración de su legado compositivo propio.

La estructura y los contenidos de estos tres Álbumes para la juventud se mantuvieron en el tiempo. Así se puede observar en Literarisches Verzeichniss der im Druck erschienenen Tonwerke von Robert Schumann, elaborado por el pianista, editor y bibliotecario Alfred Dörffel (1821-1905), que recoge la obra publicada de Schumann hasta 1871. Y esa misma estructuración se prolongó hasta el año 1875, como se refleja en la revista Musikalisches Wochenblatt en su número de 29 de octubre de 1875 (p. 558). En este medio se incluía un anuncio con el catálogo de las obras de Schumann editadas con Schuberth donde se muestra esta nueva organización. Así, los volúmenes presentados bajo el nombre Álbum para la juventud hasta el año 1875 quedan organizados, por tanto, del siguiente modo:

\footnotetext{
20 "Many women played the piano, but acceptance of their playing in public lagged behind appreciation of it at home gatherings. Although women were eventually nearly a third of all pianists performing at the prestigious Leipzig Gewandhaus between 1781 and 1881 (84 of 261), only 14 had appeared by 1830 . Nonetheless, Clara Wieck Schumann set a record there with 74 concerts, a feat most closely approached by Felix Mendelssohn's 47 concerts" (CLARK, 2008, p. 105).

21 Para una profundización sobre su intensa y extensa dedicación musical, véanse, entre otros volúmenes: Reich, 1985; Kühn, 1996; Vries, 1996; Borchard, 2015.

22 Schumann, Robert, Gesamtausgabe der Werke Robert Schumanns (Herausgeberschaft von Clara Schumann). Leipzig: Breitkupf \& Härtel, 1879-1893. La edición del Álbum para la juventud Op. 68 como parte de esta edición completa se correspondió con el volumen 5, serie VII, y vio la luz en 1887.
} 
Los otros Álbumes para la juventud de Robert Schumann

Virginia Sánchez Rodríguez

Tabla 5. Contenido de los volúmenes comercializados como Álbum para la juventud (1861-1875)

\begin{tabular}{l|l}
\hline \multicolumn{2}{c}{ Album für die Jugend comercializados por Schuberth (1861-1875) } \\
\hline Erstes Album für die Jugend & $\begin{array}{l}\text { Album für die Jugend Op. } 68 \text { (volumen I y volumen II) } \\
\text { 3 Jugend-Sonaten Op. } 118 \text { (volumen III) }\end{array}$ \\
\hline Zweites Album für die Jugend & $\begin{array}{l}\text { Klavierstücke für kleine und große Kinder Op. 85 (volumen } \\
\text { I) } \\
\text { Neun Ball-scenen Op. 109 (volumen II) }\end{array}$ \\
\hline Drittes Album für die Jugend* & $\begin{array}{l}\text { 38 Lieder-Transcriptionen für gebildete Pianisten* } \\
\text { *Los dos primeros álbumes se comercializaron a dos y } \\
\text { cuatro manos, mientras que el Tercer Álbum únicamente } \\
\text { fue editado para piano solo (dos manos). }\end{array}$ \\
\hline
\end{tabular}

Pero, en el año 1876, como se puede observar en el número de 29 de diciembre de Musikalisches Wochenblatt (p. 16), se reorganizaron los contenidos adscritos al título Album für die Jugend. En esta ocasión, se sigue hablando de Primer Álbum (Erstes Album) para la venta, exclusivamente, del original Op. 68. El Segundo Álbum (Zweites Album) ahora aparece vinculado, únicamente, al Op. 85. Por su parte, el Tercer Álbum (Drittes Album) recoge, en exclusiva, el Op. 109 -que anteriormente formaba parte del Segundo Álbum-. Pero, además, se incluye un Cuarto Álbum (Viertes Album), que contiene el Op. 118 -que anteriormente se comercializaba como parte del Primer Álbum-, e incluso un Quinto Álbum ${ }^{23}$ (Fünftes Album) que ahora está formado por las 38 Lieder-Transcriptionen -que antes configuraban el Tercer Álbum- y donde ahora Reinecke es mencionado como único responsable de la transcripción.

23 Appel (2010, p. 220) menciona, de forma escueta y anecdótica, la existencia de un Tercer y Cuarto Álbum para la juventud, pero no habla de este Quinto Álbum. 
Los otros Álbumes para la juventud de Robert Schumann

Virginia Sánchez Rodríguez

Figura 4. Drittes Album für die Jugend. Neun Ball-scenen Op. 109 (c. 1876)

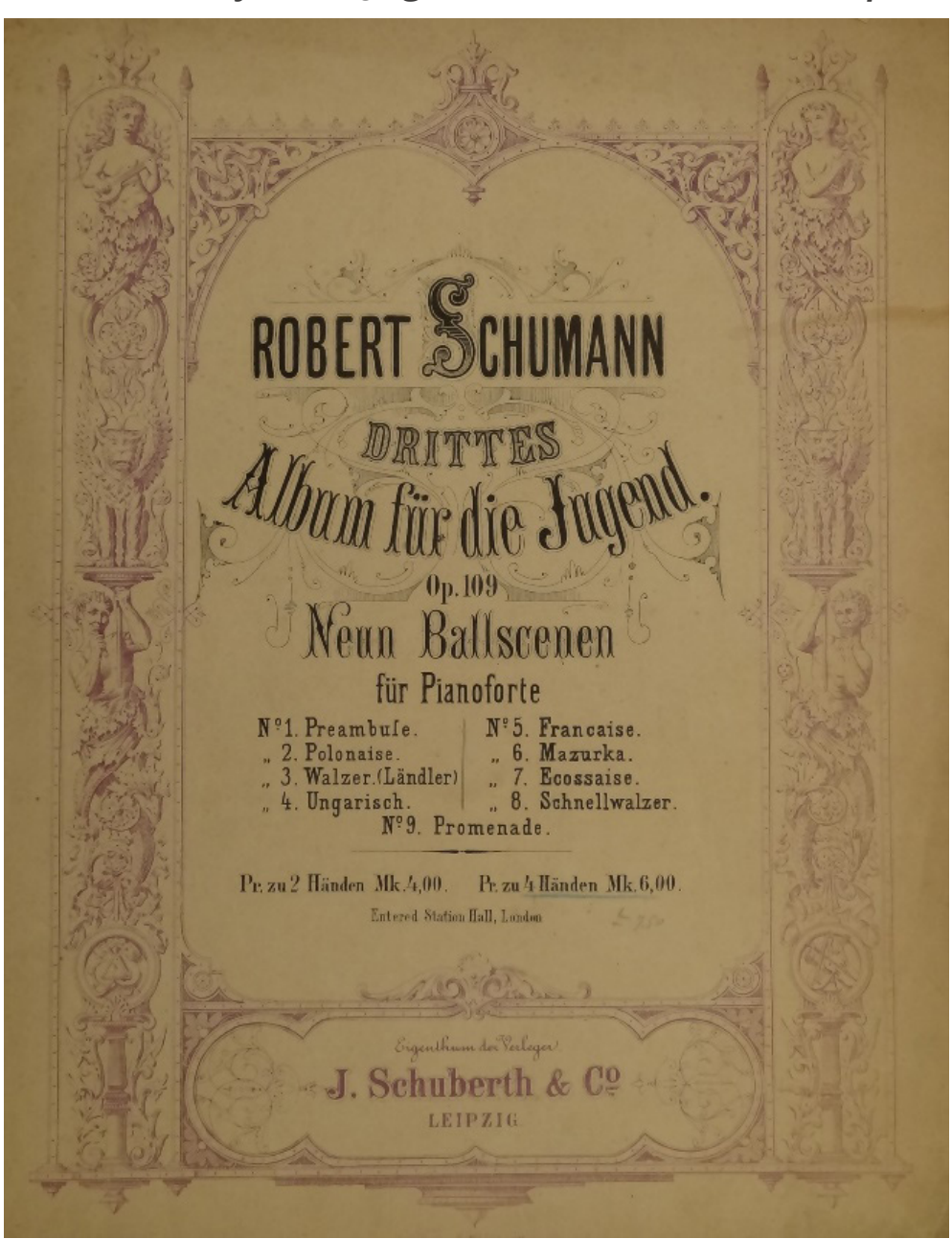

Fuente: Colección Sánchez Rodríguez (España)

Tabla 6. Contenido de los volúmenes comercializados como Álbum para la juventud (1876)

\begin{tabular}{l|l}
\hline \multicolumn{2}{c}{ Album für die Jugend comercializados por Schuberth (1876) } \\
\hline Erstes Album für die Jugend & Album für die Jugend Op. 68 (volumen I y volumen II) \\
\hline Zweites Album für die Jugend & Klavierstücke für kleine und große Kinder Op. 85 \\
\hline Drittes Album für die Jugend & Neun Ball-scenen Op. 109 \\
\hline Viertes Album für die Jugend & Zwölf grössere Klavierstücke in 3 Sonaten Op. 118. \\
\hline Fünftes Album für die Jugend & 38 Lieder-Transcriptionen für gebildete Pianisten \\
\hline & Fuente: elaboración propia.
\end{tabular}

Los cinco álbumes fueron comercializados de este modo hasta el final del siglo XIX. En el catálogo de la editorial Schuberth de 
1906 (pp. 76-77; 150-151) se puede constatar que, en esa fecha, ya no se distribuía el Quinto Álbum de la juventud. Así, a comienzos del siglo XX, Schuberth mantenía en catálogo el Primer Álbum formado solo por el Op. 68, el Segundo Álbum con el Op. 85, el Tercer Álbum conteniendo el Op. 109 y el Cuarto Álbum correspondiente al Op. 118. Estos cuatro cuadernos estaban disponibles tanto en su versión para dos manos como en la edición para cuatro manos.

En este punto podríamos plantearnos qué sucede, por tanto, con las 38 Lieder-Transcriptionen für gebildete Pianisten del Quinto Álbum. En el año 1906, éstas seguían formando parte del catálogo de Schuberth, pero con la particularidad de que ya no se presentaban bajo la marca comercial de Álbum para la juventud de Schumann sino como parte del legado del propio Carl Reinecke bajo el título Achtunddreißig Transkriptionen Schumanngeher Lieder (SCHUBERTH, 1906, p. 137) (38 transcripciones de canciones de los Schumann). Desconocemos las razones que llevaron al editor a presentar estas melodías con un mayor protagonismo de su transcriptor, que aún vivía en esa fecha. Probablemente esta nueva edición, y el protagonismo de Reinecke en la misma, podría haber estado determinada con la visibilidad del músico en la Alemania del momento, con el reconocimiento de su labor como transcriptor el propio Schumann no solo le dedicó su Op. 72, sino que confiaba plenamente en su trabajo para estas transcripciones, como se puede comprobar a través de la carta enviada a Reinecke con fecha de 30 de junio de 1848 expuesta previamente- $y$, probablemente, con el interés de Schuberth de utilizar su nombre como un nuevo reclamo en la comercialización de estas transcripciones a partir de los Lieder de Robert y Clara Schumann.

Podría sorprendernos que se continuaran distribuyendo esta serie de volúmenes bajo el título Álbum para la juventud, incluso modificando los contenidos, en una fecha tan tardía como comienzos del siglo XX y después de que Clara Schumann hubiera editado la obra completa de su esposo con Breitkopf \& Härtel a finales del siglo anterior. En todo caso, en lo que respecta a los álbumes objeto de nuestro trabajo, desconocemos la fecha 
Los otros Álbumes para la juventud de Robert Schumann

Virginia Sánchez Rodríguez

exacta en la que dejaron de comercializarse -circunstancia en la que profundizaremos en futuros trabajos-, lo que no empaña, en nuestra opinión, la visibilidad de los opus presentados y el éxito en la comercialización de esta marca comercial en la Alemania de la segunda mitad del siglo XIX.

\section{Conclusiones}

Tras todo lo comentado hasta el momento, en primer lugar, podemos corroborar el elevado valor del Álbum para la juventud de Robert Schumann por la combinación, en todas sus piezas, de nociones técnicas, un carácter pedagógico y un evidente sentido artístico. Por ello, podemos afirmar que Schumann propone una renovación de la literatura pianística de un nivel básico y medio, fomentando la visibilidad social de estas melodías en torno al concepto de Hausmusik del que nace y para el que aparece destinado. Ahora bien, de la misma forma que la esencia del Álbum para la juventud no solo se refleja en posteriores composiciones del músico alemán, la gran acogida de este Álbum fue también utilizada por Schuberth, su editor, de forma póstuma.

Después del fallecimiento de Schumann, el término Álbum para la juventud -el título original de las 42 piezas compuestas en 1848 y que comenzaron a publicarse como 43 piezas en la segunda edición de 1850- fue utilizado por el editor Julius Schuberth para la publicación de nuevas ediciones de otras obras de Robert Schumann hasta 1886, fecha en la que finalizaba el compromiso de los derechos de edición, que, según el contrato (APPEL, 2010, p. 220), se extendía durante treinta años después del fallecimiento del músico. En concreto, tal como hemos expuesto, en la segunda mitad del siglo XIX, desde 1861, existieron hasta un total de cinco Álbumes para la juventud que acogieron, a través de las diferentes organizaciones expuestas en nuestro trabajo, 12 Klavierstücke für kleine und große Kinder Op. 85, Neun Ball-scenen Op. 109, Zwölf grössere Klavierstücke in 3 Sonaten Op. 118 (publicadas originalmente 
como 3 Jugend-Sonaten) y 38 Lieder-Transcriptionen für gebildete Pianisten -siendo ésta última obra la única que, a comienzos del siglo XX, en el catálogo de la editorial Schuberth de 1906, ya aparecía desvinculada al concepto de Álbum para la juventud-.

Desconocemos las razones que llevaron al editor a publicar los mencionados opus bajo la etiqueta de Álbum para la juventud, aunque, tras lo expuesto, podemos observar que los opus expuestos comparten ciertas características, como la dignificación de la sencillez y el interés en la progresión técnica a través de melodías armónicas y con un cuidado tratamiento estético. Ahora bien, más allá de esto, consideramos que, seguramente, la utilización de esta idea por parte de Schuberth, el editor, tendría que ver con razones comerciales.

Podemos pensar que el editor apeló a la marca Álbum para la juventud, tras el éxito del Op. 68, como un reclamo publicitario para dar más salida a los restantes opus mencionados, apelando a su acercamiento a los principiantes y jóvenes músicos. Y, a tenor de las diferentes estructuraciones sufridas entre 1961 y 1976, podemos imaginar que esa estrategia habría sido efectiva, de ahí la continua propuesta y modificación de Álbumes. Igualmente, el elevado número de Álbumes para la juventud que Schuberth lanzó a partir de la reutilización de obras de Schumann también tuvo que ver con el perfil editorial de Schuberth, que se especializó en este género de música para niños. Como expone Isabel Eicker, en el fondo esta eclosión de Álbumes para la juventud de Schumann coincide con el florecimiento de álbumes para niños (EICKER, 1995). Así lo refleja Kok:

From Isabel Eicker's inventory of children's piano albums advertised in the Hofmeister Whistling catalogues, we know that between 1843 and 1891 Schuberth \& Co. published children's works by at least fifteen composers besides Schumann (KOK, 2008, p. 103). 
Queda evidenciado, por tanto, el interés de Schumann de dotar de un repertorio pianístico para una etapa tan importante de la vida, como la infancia y la juventud, y el provecho comercial extraído por parte de Schuberth, el editor de la obra, dando lugar a la creación de una marca comercial. Asimismo, además de todo lo expuesto, podemos afirmar que esta eclosión de los otros Álbumes para la juventud sirvió para la transmisión del legado de Robert Schumann y su estudio en la actualidad nos permite reflexionar sobre la importancia de las acciones editoriales en la difusión musical.

\section{Bibliografía}

Appel, Bernhard R. 'Actually, Taken Directly from Family Life': Robert Schumann's Album für die Jugend. In: TODD, R. Larry (Ed.). Schumann and His World. Princeton: Princeton University Press, 1994, pp. 171202.

Appel, Bernhard R. Robert Schumanns "Album für die Jugend." Einführung und Kommentar. 2 ed. Mainz: Schott, 2010.

Borchard, Beatrix. Clara Schumann. Ihr Leben. Eine biographische Montage. Hildesheim: Olms, 2015.

\section{Carrier, Carolyn. Memory and Commemoration in Robert}

Schumann's Album Leaves. Tesis Doctoral. Bloomington: Indiana University, 2019.

\section{Clark, Linda L. Women and Achievement in Nineteenth-Century}

Europe. Cambridge: Cambridge University Press, 2008.

Cooper, Suzanne. Clara Schumann, You Mean... Robert Schumann's Wife. In: Ostleitner, Elena y Simek, Ursula (Eds.). Ich fahre in mein liebes Wien, Clara Schumann: Fakten, Bilder, Projektionen. Viena: Löcker Verlag, 1996, pp. 151-158.

Daverio, John. Robert Schumann: Herald of a 'New Poetic Age'. Oxford y New York: Oxford University Press, 1997. 
Los otros Álbumes para la juventud de Robert Schumann

Virginia Sánchez Rodríguez

Daverio, John: Piano Works I: a world of images. In: PERREY, Beate (Ed.).

The Cambridge Companion to Schumann. Cambridge: Cambridge University Press, 2007, pp. 63-85.

Deahl, Lora. Robert Schumann's Album for the Young and the Coming of Age of Nineteenth-Century Piano Pedagogy. College Music Symposium, v. 41, pp. 25-42, 2001.

Dias de Oliveira Alcantara, Alexandre. La recepción de Jean Paul Richter en la obra temprana de Robert Schumann. Tesis Doctoral. Madrid: Universidad Autónoma de Madrid, 2017.

Eicker, Isabel. Kinderstücke. An Kinder adressierte und über das Thema der Kindheit komponierte Alben in der Klavierliteratur des 19. Jahrhunderts. Kassel: Bosse, 1995.

Eigeldinger, Jean-Jacques. Kinderszenen Op. 15 de Schumann: entre variations et cycle. Musurgia, v. 1, n. 1, pp. 53-65, 1994.

Finson, Jon W. Schumann's Mature Style and the Album of Songs for the Young. The Journal Musicology, v. 8, n. 2, pp. 227-250, 1990.

Fucci Amato, Rita de Cássia. Educaçao pianística: O rigor pedagógico dos Conservatórios. Música Hodie, v. 6, n. 1, pp. 75-96, 2006.

Geck, Martin. Robert Schumann: The Life and Work of a Romantic Composer. Chicago: The University of Chicago Press, 2013.

Gustems, Josep; Chaib, Danilo; Font, Joana; Iglesias, Clara; Miralpeix, Antoni; Oliver, Vicent; Pon, Maria Dolors; Solà, Marta; Vila, Mateu. Algunas aportaciones de Robert Schumann a la didáctica de la música. Música y educación: Revista trimestral de pedagogía musical, año 20, n. 69, pp. 49-54, 2007.

Jensen, Eric Frederick. Schumann. Oxford: Oxford University Press, 2001.

Kok, Roe-Min y Tunbridge, Laura (Eds.). Rethinking Schumann. Oxford: Oxford University Press, 2011.

Kok, Roe-Min. Negotiating Children's Music: New Evidence for Schumann's 'Charming' Late Style. Acta Musicologica, v. 80, n. 1, pp. 99-128, 2008. 
Los otros Álbumes para la juventud de Robert Schumann

Virginia Sánchez Rodríguez

Kok, Roe-Min. Of Kindergarten, Cultural Nationalism, and Schumann's Album for the Young. The World of Music, v. 48, n. 1, pp. 111-132, 2006.

Kühn, Dieter. Clara Schumann, Klavier: Ein Lebensbuch. Frankfurt am Main: Fischer, 1996.

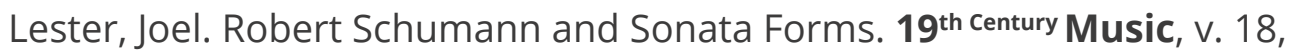
n. 3, pp. 189-210, 1995.

MacAuslan, John. Schumann's Music and E. T. A. Hoffmann's Fiction. Cambridge: Cambridge University Press, 2016.

MacDonald, Claudia. Schumann's Piano Practice: Technical Mastery and Artistic Ideal. The Journal of Musicology, v. 19, n. 4, pp. 527-563, 2002.

Marston, Nicholas. Heroes: Schubert, Beethoven, Bach. In: PERREY, Beate (Ed.). The Cambridge Companion to Schumann. Cambridge: Cambridge University Press, 2007, pp. 48-61.

Musikalisches Wochenblatt, año VI, n. 44 (29 de octubre), p. 558, 1875 Musikalisches Wochenblatt, año VIII, n. 1 (29 de diciembre), p. 16, 1876. Neue Zeitschrift für Music, v. 54, n. 8, (15 de febrero), p. 8, 1861. Neue Zeitschrift für Musik, v. 32, n. 36 (3 de mayo), pp. 1-4, 1850. Neue Zeitschrift für Musik, v. 51, n. 4, (22 de julio), p. 32, 1859. Neue Zeitschrift für Musik, v. 55, n. 15, (4 de octubre), p. 132, 1861. Neue Zeitschrift für Musik, v. 55, n. 17 (18 de octubre), p. 141, 1861. Neue Zeitschrift für Musik, v. 55, n. 7 (9 de agosto), p. 60, 1861. Newcomb, Anthony. Schumann and the Marketplace: From Butterflies to Hausmusik. In: TODD, R. Larry (Ed.). Nineteenth-Century Piano Music. New York: Schirmer, 1990, pp. 258-314.

Ostwald, Peter F. Schumann: The Inner Voices of a Musical Genius. Boston: Northeastern University Press, 1985.

Perrey, Beate (Ed.). The Cambridge Companion to Schumann. Cambridge: Cambridge University Press, 2007.

Plantinga, Leon B. Schumann as Critic. New Haven: Yale University Press, 1967. 
Los otros Álbumes para la juventud de Robert Schumann

Virginia Sánchez Rodríguez

Reich, Nancy B. Clara Schumann: The Artist and the Woman. Ithaca and London: Cornell University Press, 1985.

Rosen, Charles. Sonata Forms. New York: Norton, 1980.

Sánchez Rodríguez, Virginia. Clara Schumann and the Schwarz Family: Reconstructing a Friendship through an Edition of Robert Scuhmann's Zweites Album für die Jugend. Notes: The Quarterly Journal of the Music Library Association, vol. 77, n. 3, pp. 380-404, 2021.

Schmidt, Mathhias. 'Erinnere dich [...] unserer Zukunft': Komponierte Kindheit in Robert Schumanns Op. 15. Acta Musicologica, v. 76, fasc. 1, pp. 67-87, 2004.

Schuberth, Julius. Katalog des Musikalien-Verlags von J. Schuberth \& Co. Leipzig: J. Schuberth \& Co, 1906.

Schumann, Robert. Album para la juventud Op. 68 (Wiener Urtext Edition). Madrid: Real Musical, 1986.

Schumann, Robert. Gesamtausgabe der Werke Robert Schumanns (Herausgeberschaft von Clara Schumann). Leipzig: Breitkupf \& Härtel, 1879-1893.

Schumann, Robert. Haus-und Lebensregeln. Neue Zeitschrift für Musik, v. 32, n. 36 (3 de mayo), Beilage, pp. 1-4, 1850.

Schumann, Robert. Literarisches Verzeichniss der im Druck erschienenen Tonwerke von Robert Schumann. Leipzig: Fritzsch, [1871].

Schumann, Robert. Zweites Album für die Jugend. Leipzig: J.

Schuberth \& Comp., 1861.

Starobinski, Georges. Les Kinderszenen Op. 15 de Schumann: Composantes littéraires et biographiques d'une genèse. Revue de Musicologie, t. 88, n. 2, pp. 361-388, 2002.

Stefaniak, Alexander. Schumann's Virtuosity: Criticism, Composition, and Performance in Nineteenth-Century Germany. Bloomington: Indiana University Press, 2016.

Todd, R. Larry (Ed.). Schumann and His World. Princeton: Princeton University Press, 1994. 
Los otros Álbumes para la juventud de Robert Schumann

Virginia Sánchez Rodríguez

Turchin, Barbara. Schumann's Conversion to Vocal Music: A

Reconsideration. The Musical Quarterly, v. 67, n. 3, pp. 392-404, 1981.

Turnbridge, Laura. Schumann's Late Style. Cambridge: Cambridge University Press, 2007.

Vries, Claudia de. Die Pianistin Clara Wieck-Schumann:

Interpretation im Spannungsfeld von Tradition und Individualität.

Mainz: Schott, 1996.

Wasielewski, Wilhelm Joseph von. Life of Robert Schumann, with Letters, 1833-1852. London: William Reeves, 1878. 